\title{
Silver nanowires as flexible transparent electrode: role of PVP chain length
}

\begin{abstract}
In this project, crystalline silver nanowires (AgNWs) are successfully grown using a continuous segmented flow process. The robust relationship among the structural, electrical and optical properties of the AgNWs in the function of the polyvinylpyrrolidone (PVP) chain length is elaborated. A concise carrier transport and a density mechanism are also discussed using a localized conductive atomic force microscopy analysis. The obtained results proved that the AgNWs synthesized using PVP with a chain length of $1.3 \mathrm{M}$ exhibit excellent electrical and optical properties in the form of flexible transparent film with a sheet resistance of $<150 \Omega /$ sq and transparency of $>90 \%$ at various bending angles. These findings present an alternative approach for production of AgNWs and fabrication of a high flexible transparent electrode.
\end{abstract}

Keyword: Flexible; Flow chemistry; Silver nanowires; Transparent conductive film 